CONGENITAL HEART DISEASE

\title{
Timing of presentation and postnatal outcome of infants suspected of having coarctation of the aorta during fetal life
}

\author{
C E G Head, V C Jowett, G K Sharland, J M Simpson
}

Heart 2005;91:1070-1074. doi: 10.1136/hrt.2003.033027

\begin{abstract}
Objective: To report the timing of presentation and clinical profile of a cohort of fetuses with normal main cardiac connections but fetal echocardiographic signs suggestive of coarctation of the aorta.

Design: Retrospective observational study.

Setting: Tertiary fetal and paediatric cardiology centre.

Patients: Between 1 January 1998 and 31 December 2002, 174 fetuses were studied, of whom 144 infants were born alive.

Main outcome measures: Of the 144 liveborn infants, 43 had coarctation of the aorta, four had interruption of the aortic arch, and one was managed as having hypoplastic left heart syndrome. Hemianomalous pulmonary venous drainage was diagnosed in two infants. Three infants with coarctation presented late at 7-13 weeks of age, 6-12 weeks after closure of the arterial duct. Fetuses with cardiac asymmetry had a higher incidence of left superior vena cava than a control group. For fetuses with cardiac asymmetry, the incidence of left superior vena cava and ventricular septal defects was similar in infants who proved to have coarctation postnatally and in those who did not. The 30 day and one year surgical mortality of infants having repair of coarctation of the aorta was two of $41(4.9 \%, 95 \%$ confidence interval (CI) 0.6 to 16.0). All cause mortality of liveborn infants with any abnormality of the aortic arch was five of $48(10.4 \%, 95 \% \mathrm{Cl} 3.5$ to 22.7$)$ at 30 days and one year, which was heavily influenced by prematurity and extracardiac abnormalities.

Conclusions: Precise diagnosis of coarctation of the aorta during fetal life remains difficult. Coarctation of the aorta may present several weeks after closure of the arterial duct and sequential echocardiography is recommended.
\end{abstract}

See end of article for authors' affiliations

Correspondence to: Dr John M Simpson, Fetal Cardiology Unit,

Department of Congenital Heart Disease, Guy's Hospital, London SE1 9RT, UK; john.simpson@gstt. sthames.nhs.uk

Accepted 31 July 2004

D iagnosis of congenital heart defects by fetal echocardiography is now firmly established with large series in the literature documenting the diagnosis of a wide variety of lesions. ${ }^{1}$ Coarctation of the aorta is one of the most difficult conditions to diagnose during fetal life, primarily because of patency of the arterial duct and the parallel circulation that exists before birth. The presence of coarctation of the aorta may be suspected on fetal echocardiography by asymmetry of the great arteries, the ventricles, or both, with dominance of right heart structures. ${ }^{2}{ }^{3}$ Two large series have documented the antenatal features consistent with coarctation of the aorta, but some fetuses with antenatally suspected coarctation of the aorta prove to be normal postnatally. ${ }^{3}$

A variety of theories have been proposed for the development of coarctation of the aorta including subnormal flow around the aortic arch or that a sling of ductal tissue around the aorta causes the coarctation to develop at the time of ductal closure. ${ }^{4-6}$ Data regarding patterns of familial recurrence of congenital heart disease confirm the importance of genetic factors. ${ }^{7}$

There has been no large series in the literature to date documenting the postnatal course of infants who had fetal echocardiographic signs suggestive of coarctation of the aorta. The objective of this study was to report the outcome of such a cohort.

\section{PATIENTS AND METHODS}

Echocardiographic findings for all fetuses evaluated at our fetal cardiology unit are entered prospectively on a computerised database (Filemaker Pro V2-4, Claris Corporation, Santa Clara, California, USA). This database was searched for fetuses in whom a suspicion of coarctation of the aorta was

recorded, based on the opinion of the attending fetal cardiologist. During fetal life, coarctation of the aorta manifests as asymmetry of the great arteries or ventricles with dominance of right heart structures. Hypoplasia of the aortic arch is often gauged by comparing the size of the ductal arch with that of the aortic arch in a high, oblique view of the fetal thorax (three vessel view). ${ }^{8}$ Fetuses with such features are typically described as having cardiac "asymmetry" or "disproportion". These are the terms used in this report. For the fetal cardiologist, the degree of certainty about the diagnosis of coarctation varies given that, to date, no quantitative echocardiographic study has clearly differentiated fetuses with and without coarctation of the aorta.

For clarity, all fetuses in whom postnatal echocardiography had been recommended were included, regardless of the fetal cardiologist's level of suspicion. The unit policy throughout the study was to request postnatal echocardiography on any fetus in whom there were any suggestive echocardiographic features of coarctation of the aorta in the mid-trimester of pregnancy or earlier.

Postnatal records and postmortem reports were reviewed to determine the postnatal diagnosis, timing of presentation, and final outcome. Fetuses with abnormalities of the cardiac connections were excluded. Fetuses with an associated ventricular septal defect were included, if this was the sole associated lesion. We included data from fetuses where pregnancy ended in termination of pregnancy or spontaneous intrauterine death only if postmortem data were available to confirm or refute a diagnosis of coarctation of the aorta. No attempt was made in this study to refine fetal echocardiographic markers for coarctation of the aorta, as this is the subject of ongoing research within our unit. The outcome of 
all fetuses scanned at our unit is obtained from questionnaires returned at 6-8 weeks of age from general practitioners, parents, and referring obstetricians. Outcome data are available for over $95 \%$ of fetuses.

\section{Statistical analysis}

Where appropriate, groups were compared by Fisher's exact test.

\section{RESULTS}

Between January 1998 and December 2002, our unit recorded echocardiograms for 7264 fetuses. Of these, 271 fetuses had some degree of suspicion of coarctation of the aorta (fig 1). We excluded 86 fetuses because coarctation was diagnosed as part of complex congenital heart disease. No follow up information was available for 11 fetuses. Twenty four pregnancies were terminated and there were six intrauterine deaths; postmortem results were available for 19 of these (table 1). In six cases, hypoplasia of left heart structures was so severe that the pathologist categorised the fetuses as having variants of the hypoplastic left heart syndrome, although in all six cases the mitral and aortic valves were patent. Postmortem examination did not confirm coarctation of the aorta for three fetuses. The first had cerebral ventriculomegaly, polydactyly, multiple echogenic foci, bilateral cleft lip and palate, and a deletion on one of the $\mathrm{X}$ chromosomes. The second fetus had the BeckwithWiedemann syndrome (with a ventricular septal defect). The third had a bicuspid aortic valve with hypoplasia of the transverse aortic arch but no coarctation shelf; this fetus also had brain malformations including hypoplasia of the cerebellum and absence of the corpus callosum. There were
Table 1 Postmortem diagnoses after termination of pregnancy or intrauterine death

\begin{tabular}{lc}
\hline Postmortem diagnosis & Number \\
\hline CoA & 9 \\
Severe CoA causing hypoplasia of the & \\
left heart (hypoplastic left heart syndrome) & 6 \\
Interrupted aortic arch & 1 \\
No CoA* & 3 \\
Total & 19
\end{tabular}

*All fetuses in this group had other malformations or chromosomal abnormality.

CoA, coarctation of the aorta.

144 liveborn infants. During the study period, one baby who had undergone fetal echocardiography at our unit presented postnatally with coarctation of the aorta that had not been suspected prenatally. This fetus presented at 35 weeks' gestation with atrial flutter. There was some asymmetry between the right and left heart structures but this was attributed to the late gestation, and the associated arrhythmia made interpretation more difficult. This baby was delivered in sinus rhythm after digoxin administration for the arrhythmia and presented while in hospital with diminished femoral pulses. This baby's coarctation of the aorta was repaired through a left thoracotomy.

\section{Postnatal course of liveborn infants}

Hypoplastic left heart $(n=1)$

One baby had such severe hypoplasia of left heart structures that a biventricular repair was not judged feasible, despite patency of the mitral and aortic valves. This child is now four

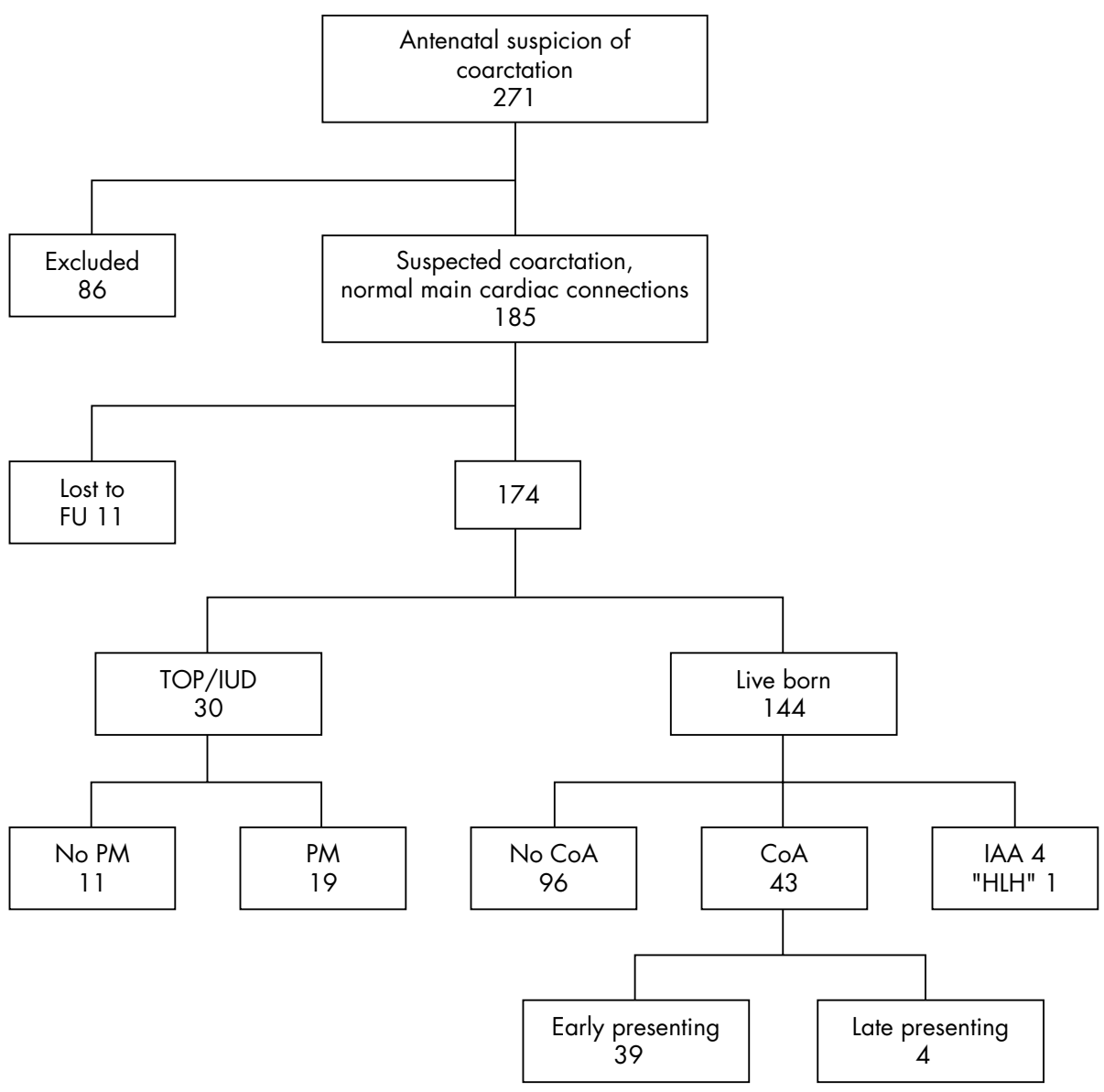

Figure 1 Flow chart of fetuses with suspected coarctation of the aorta during fetal life. CoA, coarctation of the aorta; FU, follow up; $\mathrm{HLH}$, hypoplastic left heart; IAA, interrupted aortic arch IUD, spontaneous intrauterine death; PM, postmortem examination; TOP, termination of pregnancy. 
years of age and has recently completed the third stage of Norwood surgery.

Interrupted aortic arch $(\mathrm{n}=4)$

In four infants, interruption of the aortic arch was confirmed postnatally. All of these infants had an associated ventricular septal defect. One of them had a chromosome 22qll deletion. In three of the four the arch has been successfully repaired with closure of the ventricular septal defect and are surviving (median follow up three years, range 2-5). One infant, with exomphalos diagnosed prenatally, died after repair of the aortic arch and the exomphalos.

\section{Coarctation of the aorta $(n=43)$}

Coarctation of the aorta was confirmed postnatally in 43 infants. Eleven of these infants had associated ventricular septal defects diagnosed prenatally (26\%). Three infants (7\%) had bilateral superior caval veins with the left superior vena cava draining to the coronary sinus. One infant had trisomy 21 and another had XO/XY mosaicism. Forty one infants underwent surgery at a median age of 4 days (range 1 day to 15 weeks). Twenty of the 41 infants (49\%) had hypoplasia of the aortic arch that was sufficiently severe that repair was undertaken through a median sternotomy with cardiopulmonary bypass. For the remaining infants, a lateral thoracotomy was preferred. Eleven infants had an associated ventricular septal defect, which was closed in nine infants and the pulmonary artery banded in the remaining two. The mortality of infants undergoing surgery was two of 41 ( $4.9 \%$, 95\% confidence interval (CI) 0.6 to 16.0)) at 30 days and at one year. The first baby to die had a coexistent left sided diaphragmatic hernia, which had been diagnosed prenatally and had been repaired on day 2 of life. After coarctation repair on day 9, the baby developed complications of hypoxia and pulmonary hypertension resulting in death on day 16. The second infant to die had coarctation of the aorta and aortic stenosis. The coarctation was repaired on day 6 and the baby was discharged on day 13 with good ventricular function and a pressure drop of $45 \mathrm{~mm} \mathrm{Hg}$ across the aortic valve. This infant presented again with circulatory collapse on day 28 and could not be resuscitated.

Two infants with coarctation of the aorta died without surgery. The first of these was one of twins who was delivered preterm at 24 weeks' gestational age and died of respiratory complications of prematurity. The second baby who died without surgery was delivered at 32 weeks weighing $1.3 \mathrm{~kg}$. Cardiac review at another cardiac centre was reported as "normal". This infant died suddenly on day 7. Postmortem examination reported "an anatomically narrow aortic arch" but the arterial duct was widely patent. Patchy consolidation was observed within the lung fields. For our analysis the baby is judged to have had coarctation of the aorta. Thus, on the basis of intention to treat, four of 43 (9\%, 95\% CI 2.6 to 22.0 ) liveborn infants with coarctation of the aorta died.

Of the 43 cases of confirmed coarctation, 39 (90\%) were diagnosed and treated within the first 10 days after birth.
One preterm infant (28 weeks' gestation) was electively maintained on prostaglandin $\mathrm{E}$ for 25 days until coarctation repair. Four other infants had repair of coarctation beyond 10 days of age (table 2).

The first of these infants was delivered at term but on day 13 underwent ligation of a large patent arterial duct. At surgery, a discrete coarctation was visualised and repaired. The second infant was discharged on day 6 after ductal closure: the descending aorta was pulsatile with a maximum Doppler velocity of $2.8 \mathrm{~m} / \mathrm{s}$. The findings were identical one week later but at seven weeks there were no femoral pulses, systemic hypertension, impaired left ventricular function, and a Doppler derived pressure drop of $64 \mathrm{~mm} \mathrm{Hg}$ across the coarctation site. The coarctation was repaired by end to end anastomosis at nine weeks through a left lateral thoracotomy.

The third infant was discharged from our centre on day 6 with a normal physical examination. An echocardiogram confirmed ductal closure with a maximum Doppler velocity of $1 \mathrm{~m} / \mathrm{s}$ in the descending aorta. At 6 weeks of age, femoral pulses were reduced but with good left ventricular function and a maximum Doppler velocity of $3 \mathrm{~m} / \mathrm{s}$ in the descending aorta. At 9 weeks the femoral pulses were impalpable and echocardiography showed the classical Doppler double envelope of flow into the descending aorta. Magnetic resonance imaging confirmed coarctation of the aorta with mild hypoplasia of the aortic arch. Coarctation repair was by an end to end anastomosis through a lateral thoracotomy. The fourth baby was delivered at term and the duct was closed on the second day postnatally. The femoral pulses were easily palpable at discharge on day 9. The maximum Doppler flow velocity in the descending aorta was $2 \mathrm{~m} / \mathrm{s}$. The baby was reviewed at 13 weeks: the femoral pulses were not palpable and the Doppler velocity across the coarctation site was $4.3 \mathrm{~m} / \mathrm{s}$ with reduced left ventricular systolic function. Magnetic resonance imaging showed arch hypoplasia with coarctation and an extended arch repair was performed.

\section{Infants who did not have coarctation of the aorta confirmed postnatally}

Coarctation of the aorta was not confirmed postnatally in 96 babies, of whom $20(21 \%)$ had ventricular septal defects that had been identified prenatally. Four of these infants had chromosomal abnormalities including trisomy 13, del5q32, $\mathrm{XX} / \mathrm{XO}$ mosaicism, and trisomy 16 mosaicism. Thirteen of the 96 (14\%) infants had persistence of a left sided superior vena cava draining to the coronary sinus. Abnormalities of the pulmonary venous drainage was confirmed postnatally in two babies. The first of these, with Scimitar syndrome, has been reported on previously. ${ }^{9}$ This child is now 4 years of age having had coil embolisation of the arterial collateral and surgical repair of hemianomalous pulmonary venous drainage.

The second baby was delivered at term. Identification of the pulmonary venous drainage was technically difficult prenatally. The initial neonatal echocardiograms were

Table 2 Summary of clinical and echocardiographic features of infants who presented beyond the age of 10 days

\begin{tabular}{llllll}
\hline Patient & Duct closure & $\begin{array}{l}\text { Confirmation } \\
\text { of CoA }\end{array}$ & $\begin{array}{l}\text { Imaging } \\
\text { modality }\end{array}$ & Age at surgery & $\begin{array}{l}\text { Outcome at last } \\
\text { follow up (age) }\end{array}$ \\
\hline 1 & Ligated day 13 & Day 13 & NA & 13 days & Well (18 months) \\
2 & Day 5 & 7 weeks & Echo & 9 weeks & Well (9 months) \\
3 & Day 6 & 9 weeks & Echo MRI & 11 weeks & Well (2 years) \\
4 & Day 2 & 13 weeks & Echo MRI & 15 weeks & Well (4 years) \\
\hline
\end{tabular}

Echo, echocardiography; MRI, magnetic resonance imaging; NA, not available. 
reported as normal but on review at three months the infant was found to be tachypnoeic. Echocardiography at that time confirmed a dilated right heart with right hemianomalous pulmonary venous drainage. This baby had an effective surgical repair and has had an uncomplicated course.

\section{Comparison of fetuses with and without coarctation of the aorta \\ Incidence of ventricular septal defects and left superior vena cava \\ For these comparisons, pregnancies resulting in liveborn} infants and termination of pregnancy or intrauterine death with postmortem results were included. Fetuses with severe hypoplasia of left heart structures or interruption of the aortic arch were also included. For a control group we also searched our database for fetuses with a persistent left superior vena cava in the absence of any abnormal cardiac findings (including cardiac asymmetry) or extracardiac abnormalities.

Twenty one of $99(21 \%)$ fetuses with cardiac asymmetry but without confirmed aortic arch abnormalities had a ventricular septal defect compared with 15 of 64 (23\%) fetuses with confirmed abnormalities of the aortic arch $(p=0.84$, Fisher's exact test). Of the 99 fetuses without coarctation of the aorta, 13 (13\%) had a persisting left sided superior vena cava compared with six of $64(9 \%)$ fetuses with aortic arch abnormalities ( $\mathrm{p}=0.62$, Fisher's exact test). Over the same study period seven of $5580(0.12 \%, 95 \%$ CI 0.03 to $2.2 \%$ ) fetuses had bilateral superior caval veins in the absence of cardiac asymmetry, congenital heart defects, or extracardiac abnormality (normal group). The incidence of a persistent left superior vena cava is significantly higher in fetuses who prove to have coarctation of the aorta than in the normal comparison group $(p<0.001)$. The same is true of fetuses with cardiac asymmetry who do not prove to have coarctation of the aorta compared with the normal group $(\mathrm{p}<0.001)$.

\section{DISCUSSION}

The data we report confirm that coarctation of the aorta remains a difficult diagnosis to make with a high degree of certainty during fetal life. One previous report confirmed the benefits of prenatal diagnosis of coarctation of the aorta in terms of reduced morbidity and improved survival. ${ }^{10}$ Unfortunately, to date, there are no quantitative fetal parameters that clearly differentiate, among fetuses with cardiac asymmetry, between those who will definitely develop coarctation of the aorta and those who will prove to be normal. ${ }^{2}$ Among pregnancies that were terminated, the vast majority of fetuses had definite abnormalities of the aortic arch. Pathologists classified some abnormalities as hypoplastic left heart by confirming the severe degree of left heart hypoplasia in this subgroup and the overlap between severe coarctation of the aorta and more classic forms of hypoplastic left heart syndrome. ${ }^{11}$ Some fetuses with coarctation of the aorta diagnosed prenatally proved to have interruption of the aortic arch. This may be due to technical difficulty imaging the distal aortic arch during fetal life and to antegrade flow in the arterial duct making differentiation difficult.

A large number of diagnoses of coarctation of the aorta were false positive in liveborn infants. This largely reflects our unit policy to recommend postnatal echocardiography for any fetus with cardiac asymmetry in the mid trimester or earlier, even where such asymmetry is mild, accepting that a significant number of these babies will not prove to have coarctation of the aorta at follow up. A careful explanation to expectant parents is required, including the need for sequential echocardiography.
Of the infants in our series with confirmed coarctation of the aorta, three babies did not develop clinical or echocardiographic signs of coarctation until 6-12 weeks after closure of the arterial duct. This confirms that imaging of the aortic arch soon after closure of the arterial duct is insufficient to exclude coarctation of the aorta. One of the suggested mechanisms of formation of coarctation of the aorta is that ductal tissue extends into the distal aortic arch, constricting the aortic arch like a sling as the arterial duct closes, with intimal proliferation playing a part later in life. ${ }^{56}$ In some infants, it is clear that constriction of the aortic arch takes several weeks to become clinically apparent. Abnormalities of pulmonary venous drainage were diagnosed postnatally in two infants who manifested asymmetry of the ventricles and great arteries during fetal life. Cardiac asymmetry is well recognised in fetuses with abnormalities of pulmonary veins, reflecting volume overload of right heart structures. ${ }^{12}$ The recognition of pulmonary venous abnormalities as well as late presenting coarctation led to our adoption of a policy to review all babies who had cardiac asymmetry during fetal life until they are around 1 year of age. It has been postulated that the presence of a persistent left superior vena cava may increase the likelihood of coarctation of the aorta. ${ }^{13}$ Our fetuses with cardiac asymmetry had a higher incidence of a left superior vena cava than did a control group of fetuses. However, for fetuses with cardiac asymmetry the presence of a left superior vena cava did not help distinguish between true positive and false positive cases. Persistence of a left superior vena cava may cause cardiac asymmetry by obstructing inflow into the left ventricle. ${ }^{14}$ Other possible explanations may be alterations of flow dynamics as the inferior vena cava enters the right atrium. If a left superior vena cava enters the coronary sinus then flow directed towards the lateral wall of the right atrium would increase. This may reduce right to left flow of blood across the foramen ovale leading to relative volume loading of the right heart. The association of isolated ventricular septal defects with cardiac asymmetry remains obscure, particularly in view of equal right and left heart pressures during fetal life. ${ }^{15}$

Almost half of the infants who proved to have coarctation of the aorta required cardiopulmonary bypass for surgical repair, reflecting the high incidence of hypoplasia of the aortic arch in infants who present during fetal life. The operative mortality of our cohort for coarctation repair is similar to that (one of 91) reported to the UK Central Cardiac Audit Database in the year 2000/2001. ${ }^{16}$ The higher overall mortality of our cohort, based on intention to treat, was heavily influenced by the presence of extracardiac abnormalities or preterm delivery.

In summary, the data we report support our current policy of at least one year's follow up of infants with suspected coarctation of the aorta during fetal life. An increased incidence of left superior vena cava was observed in fetuses with cardiac asymmetry but did not differentiate between the presence and absence of coarctation of the aorta.

\section{ACKNOWLEDGEMENTS}

We acknowledge the contribution of all staff in the Department of Congenital Heart Disease at Guy's Hospital who contributed to the management of these patients.

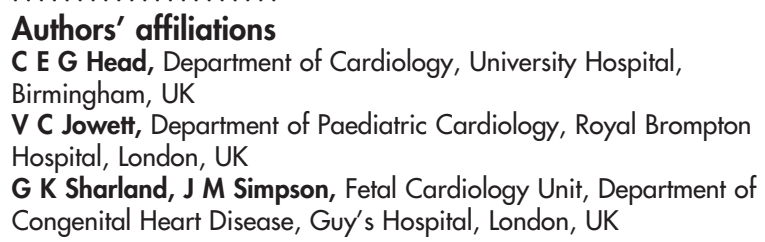




\section{REFERENCES}

1 Allan LD, Sharland GK, Milburn A, et al. Prospective diagnosis of 1006 consecutive cases of congenital heart disease in the fetus. J Am Coll Cardiol 1994;23:1452-8.

2 Sharland GK, ChanKit-Yee, Allan LD. Coarctation of the aorta: difficulties in prenatal diagnosis. Br Heart J 1994;71:70-5.

3 Hornberger LK, Sahn DJ, Kleinman CS, et al. Antenatal diagnosis of coarctation of the aorta: a multicentre experience. J Am Coll Cardiol 1994;23:417-23

4 Hornberger LK. Aortic arch anomalies. In: Lindsey A, Lisa H, Gurleen S, eds. Textbook of fetal cardiology. London: Greenwich Medical Media, 2000:307-31

5 Ho SY, Anderson RH. Coarctation, tubular hypoplasia and ductus arteriosus: histological study of 35 specimens. Br Heart $\mathrm{J}$ 1979;41:268-74.

6 Elzenga NJ, Gittenberger de Groot AC. Localised coarctation of the aorta: an age dependent spectrum. Br Heart J 1983:49:317-23.

7 Gill HK, Splitt M, Sharland GK, et al. Patterns of recurrence of congenital heart disease: an analysis of 6,640 consecutive pregnancies evaluated by detailed fetal echocardiography. J Am Coll Cardiol 2003;42:923-9.

8 Yagel S, Cohen SM, Achiron R. Examination of the fetal heart by five shortaxis views: a proposed screening method for comprehensive cardiac evaluation [editorial]. Ultrasound Obstet Gynecol 2001;17:367-9.
9 Michailidis G, Simpson JM, Tulloh RMRT, et al. Retrospective prenata diagnosis of scimitar syndrome aided by three-dimensional power Doppler echocardiography. Ultrasound Obstet Gynecol 2001;17:449-52.

10 Franklin O, Burch M, Manning N, et al. Prenatal diagnosis of coarctation of the aorta improves survival and reduces morbidity. Heart 2002;87:67-9.

11 Simpson JM. Hypoplastic left heart syndrome. Ultrasound Obstet Gynecol 2000;15:271-8.

12 Allan LD, Sharland GK. The echocardiographic diagnosis of totally anomalous pulmonary venous connection in the fetus. Heart $2001 ; 85: 433-7$

13 Agnoletti G, Annecchino F, Preda L, et al. Persistence of the left superior caval vein: can it potentiate obstructive lesions of the left ventricle? Cardiol Young 1999;9:285-90

14 Cochrane AD, Marath A, Mee RBB. Can a dilated coronary sinus produce left ventricular inflow obstruction? An unrecognised entity. Ann Thorac Surg 1994;58:1114-6.

15 Johnson P, Maxwell DJ, Tynan MJ, et al. Intracardiac pressures in the human fetus. Heart 2000;84:59-63.

16 Cunningham AD. Procedures for congenital heart disease: interim report 2000/2001-3rd draft. 31 January 2001. www.scts.org/file/Paed 20002001 pdf (accessed 7 December 2004).

\section{IMAGES IN CARDIOLOGY}

doi: $10.1136 /$ hrt. 2004.049973

\section{Uncommon anomalous papillary muscle/chordae tendineae incidentally found in patient with transient ischaemic attack}

A nomalies of mitral subvalvar apparatus include various types of anomalies of papillary muscles and chordae tendineae. Direct insertion into the anterior mitral leaflet and fusion to the ventricular septum of the anomalous papillary muscle or chordae tendineae are common types of these anomalies. Anomalous papillary muscles or chordae, especially those that are inserted directly into the mitral leaflets, play a role in augmenting left ventricular outflow obstruction by restricting mobility of the leaflets and/or tethering them toward the septum, thus narrowing the left ventricular outflow tract.

An uncommon anomalous papillary muscle/chordae was incidentally found in a 38 year old woman with transient ischaemic attack, who underwent transthoracic and transoesophageal echocardiography to evaluate a possible embolic source including patent foramen ovale. Transthoracic echocardiography showed the thick anomalous chordae tendineae was arising from the accessory papillary muscle at the anterior free wall, and inserting into the left ventricular outflow septum, without involving the mitral leaflet (panels A-C). The mitral valve leaflet or annulus were not involved in this anomalous apparatus and there was no left ventricular outflow obstruction throughout the cardiac cycle. Patent foramen ovale was not found by agitated saline contrast, and there was no sign of an intracardiac embolic source in this patient. The neurologist decided to initiate anticoagulation treatment and the patient has been doing well without further vascular events, although the relation between the cerebral vascular event and this uncommon anomalous subvalvar apparatus is not clear.
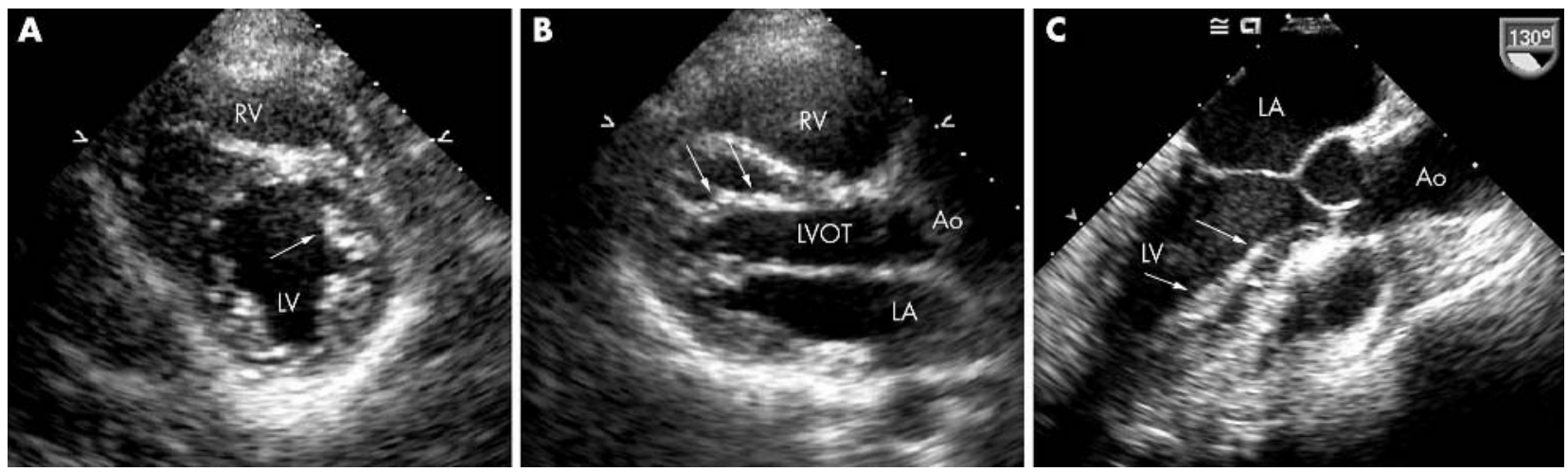

Transthoracic (TTE) and transoesophageal (TOE) echocardiograms. (A) Parasternal short axis view. Accessory papillary muscle is seen at the left ventricular anterior free wall, just anterior to the anterolateral papillary muscle (arrow). (B) Parasternal modified long axis view by TTE. (C) Long axis view by TOE. Thick anomalous chordae tendineae (arrows) arises from the accessory papillary muscle and attaches to the left ventricular outflow septum after crossing the left ventricle without creating left ventricular oufflow obstruction. Ao, aorta; LA, left atrium; LV, left ventricle; LVOT, left ventricular oufflow tract; RV, right ventricle. 